\title{
Structural Effects on the Norrish Type I $\alpha$-Bond Cleavage of Tropospherically Important Carbonyls
}

\author{
Keiran N. Rowell, ${ }^{\dagger}$ Scott H. Kable, ${ }^{\dagger}$ and Meredith J. T. Jordan ${ }^{*, \ddagger}$ \\ $\dagger$ School of Chemistry, University of New South Wales, Sydney, New South Wales, 2052, \\ Australia \\ $\ddagger$ School of Chemistry, University of Sydney, Sydney, New South Wales, 2006, Australia \\ E-mail: meredith.jordan@sydney.edu.au \\ Phone: +6129351 4420. Fax: +61293513329
}

\section{Supporting Information}

S1 Natural Transition Orbitals of Representative Carbonyls

S2 Calculated and Experimental $T_{1}$ Photolysis Thresholds

S3 The Resolution of Identity Methods Used

S4 bt-PNO-STEOM-CCSD Thresholds and Active Space

S5 Multireference Diagnostics

S6 $T_{2}-T_{1}$ Energy Separation and $\mathcal{T}_{1}$ Diagnostics

S7 Asymptotic Energy Protocols

S8 Additional 0-0 Electronic Excitation Energies, NTI Photolysis Thresholds and Intrinsic Barriers.

S9 Comparison of Calculated and Experimental Geometries

S10 Cartesian Coordinates of all Stationary Points - provided in a .zip folder 


\section{S1. Natural Transition Orbitals of Representative Car- bonyls}

The atmospheric photochemistry of carbonyls involves $n \rightarrow \pi^{*}$ excitation of an oxygen lone pair electron $(n)$ to the $\pi^{*}$ lowest unoccupied molecular orbital of the $\mathrm{C}=\mathrm{O}$ chromophore. At actinic energies, the $S_{1}$ electronic excited state is typically bound and photolysis occurs only after intersystem crossing (ISC) to $T_{1}$. The carbonyl $n$ and $\pi^{*}$ molecular orbitals are approximately orthogonal, as shown in Figure $\mathrm{S} 1$, and $T_{1} \rightarrow S_{0}$ ISC is "spin-orbit" allowed because the change in electron spin angular momentum can be accompanied by a change in electron orbital angular momentum. ${ }^{1}$ If the $T_{1}$ state is bound or if $T_{1} \rightarrow S_{0}$ ISC is competitive with $T_{1}$ photolysis, ground state reactions also need to be considered for these small carbonyls.

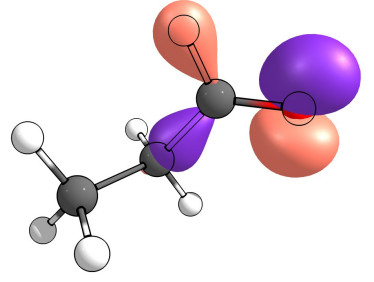

(a) propanal $n$

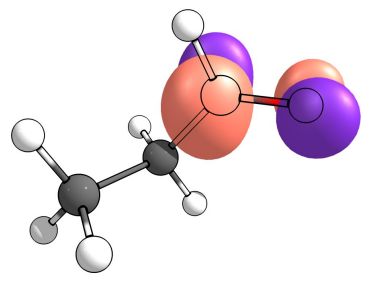

(e) propanal $\pi^{*}$

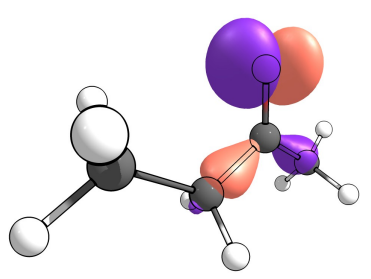

(b) butanone $n$

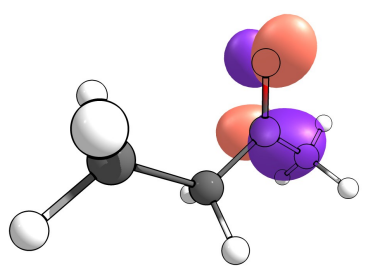

(f) butanone $\pi^{*}$

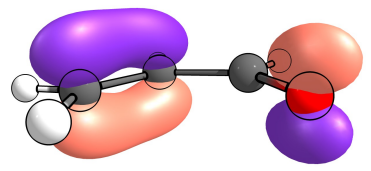

(c) acrolein $n$

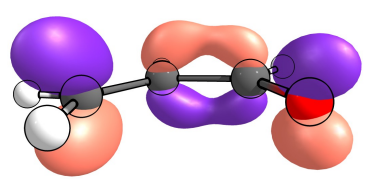

(g) acrolein $\pi^{*}$

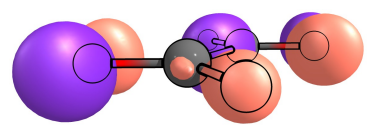

(d) glyoxal $n$

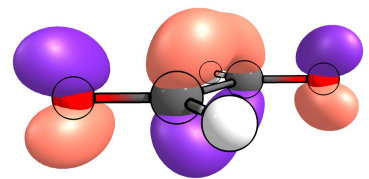

(h) glyoxal $\pi^{*}$

Figure S1: Natural transition orbital ${ }^{2}$ representations of the computed $n$ (top) and $\pi^{*}$ (bottom) orbitals for select carbonyls from the dataset. 


\section{S2. Calculated and Experimental $T_{1}$ Photolysis Thresholds}

$T_{1}$ energetic thresholds have been calculated using the unrestricted B2GP-PLYP double hybrid density functional ${ }^{3,4}$ with the empirical D3(BJ) ${ }^{5,6}$ dispersion correction, within the resolution of the identity (RI) formalism, ${ }^{7}$ and employed the def2-TVZP basis set. ${ }^{8}$ Table S1 compares our calculations to available experimental thresholds, ${ }^{9-13}$ and selected literature calculations. ${ }^{11,14-20}$ Previously calculated UM06-2X thresholds ${ }^{16}$ are also included.

Table S1 Calculated and experimental $T_{1}$ photolysis thresholds $(\mathrm{kJ} / \mathrm{mol})$.

\begin{tabular}{|c|c|c|c|c|}
\hline & B2GP-PLYP $^{a}$ & Lit. Expt. & Lit. Theory & $\mathrm{UUM}_{\mathrm{UM}} \mathrm{N}^{b}$ \\
\hline$\overline{\mathrm{HCHO}} \longrightarrow \mathrm{H} \cdot+\mathrm{CH}_{3} \mathrm{CO}$. & 378 & $385^{c}$ & $383^{d}$ & - \\
\hline $\mathrm{CH}_{3} \mathrm{CHO} \longrightarrow \mathrm{H} \cdot+\mathrm{CH}_{3} \mathrm{CO} \cdot$ & 398 & - & - & 401 \\
\hline $\mathrm{CH}_{3} \mathrm{CHO} \longrightarrow \mathrm{HCO} \cdot+\mathrm{CH}_{3}$. & 377 & $378^{e}$ & $376^{f}$ & 378 \\
\hline $\mathrm{CH}_{3} \mathrm{CH}_{2} \mathrm{CHO} \longrightarrow \mathrm{H} \cdot+\mathrm{CH}_{3} \mathrm{CH}_{2} \mathrm{CO}$. & 394 & - & $408^{g}$ & 402 \\
\hline $\mathrm{CH}_{3} \mathrm{CH}_{2} \mathrm{CHO} \longrightarrow \mathrm{HCO} \cdot+\mathrm{CH}_{3} \mathrm{CH}_{2}$. & 360 & $366^{h}$ & $364^{i}$ & 365 \\
\hline $\mathrm{CH}_{3}\left(\mathrm{CH}_{2}\right)_{2} \mathrm{CHO} \longrightarrow \mathrm{H} \cdot+\mathrm{CH}_{3}\left(\mathrm{CH}_{2}\right)_{2} \mathrm{CO}$. & 398 & - & $370^{j}$ & 405 \\
\hline $\mathrm{CH}_{3}\left(\mathrm{CH}_{2}\right)_{2} \mathrm{CHO} \longrightarrow \mathrm{HCO} \cdot+\mathrm{CH}_{3}\left(\mathrm{CH}_{2}\right)_{2} \cdot$ & 365 & - & $338^{j}$ & 364 \\
\hline $\mathrm{CH}_{3}\left(\mathrm{CH}_{2}\right)_{3} \mathrm{CHO} \longrightarrow \mathrm{H} \cdot+\mathrm{CH}_{3}\left(\mathrm{CH}_{2}\right)_{3} \mathrm{CO}$. & 398 & - & $372^{j}$ & 403 \\
\hline $\mathrm{CH}_{3}\left(\mathrm{CH}_{2}\right)_{3} \mathrm{CHO} \longrightarrow \mathrm{HCO} \cdot+\mathrm{CH}_{3}\left(\mathrm{CH}_{2}\right)_{3}$. & 365 & - & $353^{j}$ & 362 \\
\hline$\left(\mathrm{CH}_{3}\right)_{2} \mathrm{CO} \longrightarrow \mathrm{CH}_{3} \cdot+\mathrm{CH}_{3} \mathrm{CO}$ & 385 & $391^{k}$ & $403^{l}$ & 393 \\
\hline $\mathrm{CH}_{3} \mathrm{CH}_{2}(\mathrm{CO}) \mathrm{CH}_{3} \longrightarrow \mathrm{CH}_{3} \cdot+\mathrm{CH}_{3} \mathrm{CH}_{2} \mathrm{CO}$. & 393 & - & $403^{m}$ & 394 \\
\hline $\mathrm{CH}_{3} \mathrm{CH}_{2}(\mathrm{CO}) \mathrm{CH}_{3} \longrightarrow \mathrm{CH}_{3} \mathrm{CH}_{2} \cdot+\mathrm{CH}_{3} \mathrm{CO}$. & 388 & - & $391^{m}$ & 388 \\
\hline $\mathrm{CH}_{3}\left(\mathrm{CH}_{2}\right)_{2}(\mathrm{CO}) \mathrm{CH}_{3} \longrightarrow \mathrm{CH}_{3} \cdot+\mathrm{CH}_{3}\left(\mathrm{CH}_{2}\right)_{2} \mathrm{CO}$. & 400 & - & $378^{j}$ & 393 \\
\hline $\mathrm{CH}_{3}\left(\mathrm{CH}_{2}\right)_{2}(\mathrm{CO}) \mathrm{CH}_{3} \longrightarrow \mathrm{CH}_{3}\left(\mathrm{CH}_{2}\right)_{2} \cdot+\mathrm{CH}_{3} \mathrm{CO} \cdot$ & 386 & - & $367^{j}$ & 382 \\
\hline$(\mathrm{CHO})_{2} \longrightarrow \mathrm{H} \cdot+\mathrm{CHO}-\mathrm{CO}$ & 389 & - & $379^{n}$ & - \\
\hline$(\mathrm{CHO})_{2} \longrightarrow \mathrm{HCO} \cdot+\mathrm{HCO}$ & 294 & $303^{\circ}$ & $311^{n}$ & — \\
\hline MAD w.r.t Expt & 5.8 & & 5.2 & 1.0 \\
\hline MaxAE w.r.t Expt & 9 & & 12 & 2 \\
\hline
\end{tabular}

${ }^{a}$ URI-B2GP-PLYP/def2-TZVP for all optimsations, ZPVEs, single-point energies; ${ }^{b}$ UM06-2X/6$311+\mathrm{G}(3 \mathrm{df}, 2 \mathrm{p}) / / \mathrm{UB} 3 \mathrm{LYP} / 6-31+\mathrm{G}(\mathrm{d}, \mathrm{p}) ;{ }^{16} \quad c \quad$ Ref. $9 ; \quad d \quad \mathrm{UCCSD}(\mathrm{T}) /$ aug-cc-pVTZ; ${ }^{14} \quad e$ Ref. 10; $f \quad \mathrm{MRMP} / \mathrm{TZV}(2 \mathrm{df}, 2 \mathrm{p}) / / \mathrm{CAS}(10,10) /$ cc-PVDZ; $^{15} \quad g$ ROCCSD(T)/aug-cc-pVTZ//ROBH\&HLYP/6$31+\mathrm{G}(\mathrm{d}, \mathrm{p}) ;{ }^{16}{ }^{h}$ Ref. $11 ;{ }^{i} \operatorname{CCSD}(\mathrm{T}) /$ aug-cc-pVTZ//M062X/aug-cc-pVTZ; ${ }^{11}{ }^{j} \quad$ XMCQDPT2 $/ 6-$ $311++\mathrm{G}^{* *} / / \mathrm{CASSCF}(8,7) / 6-31+\mathrm{G}^{*}$ for butanal, XMCQDPT2 $/ 6-31+\mathrm{G}^{*} / \mathrm{CASSCF}(8,7) / 6-31+\mathrm{G}^{*}$ for all others; ${ }^{17}{ }^{k}$ Ref. $12 ;{ }^{l}$ (8e,7o)-CASPT2 $/ 6-31+\mathrm{G}^{*}$ (no ZPVE correction); ${ }^{18} \mathrm{~m}$ (8e,7o)-CASPT2/6-31G* (no ZPVE correction); ${ }^{19}{ }^{n}$ MR-CISD $(12,10) /$ cc-pVDZ//CAS(14,11)/cc-pVDZ; ${ }^{20}{ }^{o}$ Ref. 13.

Table S1 also shows the mean absolute deviation (MAD) and maximum absolute error (MaxAE) of the calculated $T_{1}$ thresholds, with respect to the literature experimental results, where the CASPT2 calculations of Maeda et al. ${ }^{21}$ and Nádasdi et al. ${ }^{19}$ which do not include zero-point vibrational energy (ZPVE) corrections, have not been included. Our results are consistent with, to within $10 \mathrm{~kJ} / \mathrm{mol}$, previous experiment and theory. 


\section{S3. The Resolution of Identity Methods Used}

The resolution of the identity (RI) approach was used to speed up electronic structure calculations by a factor of $\sim 2.5$, with greater efficiency gains for the larger carbonyls. All density functional theory (DFT) calculations employed the RI-JK scheme, which is efficient for "smaller" molecules. ${ }^{7}$ Auxiliary basis sets derived from the def2-TZVP canonical basis were used when required: def2 $/ \mathrm{JK}^{22}$ for the exchange and Coulomb integrals and def2-TZVP $/ \mathrm{C}^{23}$ for the correlation energy. The RI-JK scheme has not been implemented in ORCA for time dependent (TD) TD-DFT methods. Instead, the RIJCOSX scheme ${ }^{7}$ was used for all $S_{1}$ TD-DFT calculations.

\section{S4. $b t$-PNO-STEOM-CCSD Thresholds and Active Space}

Since a large cc-pVQZ canonical and auxiliary basis sets are used in these calculations, integration grids were increased to their finest pre-defined settings ('Grid7' , 'GridX7' in ORCA) and radial integration was manually set an even finer grid ('IntAcc 6.0' in ORCA). Within the bt-PNO-STEOM-CCSD approximation, the cut-off between pair natural orbitals (PNOs) was set to the most accurate threshold ('TightPNO' in ORCA). Automated active space selection was used ${ }^{24}$ and all calculations reported have an orbital percentage active character value of $>98.0$, indicating the validity of the active space.

\section{S5. Multireference Diagnostics}

The $\mathcal{T}_{1}$ diagnostic ${ }^{25}$ was calculated for optimised $S_{0}$ and $T_{1}$ stationary points using Gaussian 09 at the $\mathrm{CCSD}^{26}$ /def2-TZVP and RO-CCSD 27 /def2-TZVP levels of theory, respectively, with values reported in Table S2. As initially proposed, ${ }^{25}$ the $\mathcal{T}_{1}$ value indicating the wavefunction is well described by a single reference was taken to be 0.02 for all closed shell species. For open

shell species, following literature precedent, the acceptable $\mathcal{T}_{1}$ was deemed to be $0.044 .^{28,29}$ 
Table S2 B2GP-PLYP $T_{2}-T_{1}$ energy separation $\left(\mathrm{kJ} / \mathrm{mol}\right.$ ), and $\mathcal{T}_{1}$ ROCCSD $/$ CCSD diagnostics, for various $S_{0}$ and $T_{1}$ stationary points of select carbonyls in the dataset.

\begin{tabular}{|c|c|c|c|c|c|c|c|c|c|}
\hline & \multicolumn{3}{|c|}{$T_{2}-T_{1}$ separation } & \multicolumn{3}{|c|}{$\mathcal{T}_{1}$ diagnostic on $T_{1}$} & \multicolumn{3}{|c|}{$\mathcal{T}_{1}$ diagnostic on $S_{0}$} \\
\hline & Minima & NTI $a$ TS & NTI $b$ TS & Minima & NTI $a$ TS & NTI $b$ TS & Minima & NTI $a$ TS & NTI $b$ TS \\
\hline Glycoladehyde & 241 & 20 & 121 & 0.024 & 0.027 & 0.026 & 0.017 & 0.017 & 0.024 \\
\hline Acrolein & 314 & 35 & 97 & 0.030 & 0.028 & 0.031 & 0.020 & 0.017 & 0.023 \\
\hline Methacrolein & 306 & 56 & 69 & 0.029 & 0.025 & 0.027 & 0.018 & 0.016 & 0.021 \\
\hline Crotonaldehyde & 315 & 63 & 76 & 0.028 & 0.025 & 0.027 & 0.018 & 0.016 & 0.020 \\
\hline MVK & 305 & 60 & 29 & 0.025 & 0.026 & 0.030 & 0.020 & 0.016 & 0.017 \\
\hline MIPK & 283 & 78 & 27 & 0.019 & 0.024 & 0.028 & 0.033 & 0.015 & 0.016 \\
\hline Glyoxal & 120 & 93 & 137 & 0.026 & 0.029 & 0.030 & 0.017 & 0.020 & 0.026 \\
\hline \multirow[t]{3}{*}{ Methylglyoxal } & 89 & 105 & 104 & 0.039 & 0.027 & 0.027 & 0.016 & 0.019 & 0.024 \\
\hline & & & & Mean & 0.027 & & Mean & 0.019 & \\
\hline & & & & Max & 0.039 & & $\operatorname{Max}$ & 0.033 & \\
\hline
\end{tabular}

For $S_{0}$ carbonyls, the vast majority of $\mathcal{T}_{1}$ values were $<0.02$. The minimum energy geometry of methyl isopropenyl ketone (MIPK) and geometries corresponding to the NTI $b$ TSs of glycolaldehyde, acrolein, methacrolein, methylglyoxal and glyoxal, however, had slightly larger $\mathcal{T}_{1}$ values. Of these 6 stationary points the largest $\mathcal{T}_{1}$ value obtained was 0.033 . As this is still significantly below a threshold of $\mathcal{T}_{1} \sim 0.06$, where multireference issues are known to seriously compromise results, ${ }^{30}$ the DFT calculations of these species were not corrected for multireference character. Notably, DFT calculations are known to be less sensitive than wavefunction methods to small amounts of multireference character, ${ }^{31}$ alongside being less sensitive to spin-contamination. ${ }^{32}$

For open-shell species the mean $\mathcal{T}_{1}$ value was found to be 0.027 , with a maximum of 0.039 for the $T_{1}$ minimum energy geometry of methylglyoxal. These values are all under the 0.044 threshold, indicating single reference methods are appropriate for these species.

\section{S6. $T_{2}-T_{1}$ Energy Separation and $\mathcal{T}_{1}$ Diagnostics}

The $T_{2}-T_{1}$ energy separation and $\mathcal{T}_{1}$ diagnostics for carbonyl $S_{0}$ and $T_{1}$ stationary points are also shown in Table S2. Electronic energy separations were calculated at the TD-RI-UB3LYPD3(BJ)/def2-TZVP level of theory, where 10 roots were determined to ensure convergence of the $T_{2}$ energies. The $\mathcal{T}_{1}$ diagnostics ${ }^{25}$ were determined at the RO-CCSD/def2-TZVP and 
CCSD/def2-TZVP on the $T_{1}$ and $S_{0}$ electronic states, respectively. The mean and maximum values of the $\mathcal{T}_{1}$ diagnostics are also reported for each electronic state.

\section{S7. Asymptotic Energy Protocols}

Asymptotic product energies were calculated using two protocols to test whether more convenient optimisations of separate radical products suffered degraded accuracy due to basis set superposition error. The first protocol used separate optimisations for the two $D_{0}$ radical products. In the second protocol, a constrained supermolecular optimisation was performed on the $T_{1}$ state with the dissociating bond fixed at $20 \AA$. The $T_{1}$ products correlate to $S_{0}$ products but the $T_{1}$ wavefunction is typically more stable to singlet-triplet instability. In supermolecular calculations small $\left(<30 \mathrm{~cm}^{-1}\right)$ interfragment real and/or imaginary frequencies were encountered, which were excluded from the ZPVE. Counterpoise corrections ${ }^{33}$ failed to improve the accuracy of the $E^{\text {asymptotic }}$ values and are not included in the energies quoted below.

The thermochemical data available for our carbonyl dataset are limited. It is only possible to predict $\Delta H_{\text {react }}(0 \mathrm{~K})$ from heats of formation given in the Active Thermochemical Tables $(\mathrm{ATcT})^{34}$ for 7 of the $20 S_{0}$ NTI $a$ reactions and only a single $S_{0}$ NTI $b$ reaction. From the data in Table S3, the "separate" calculation of $D_{0}$ radicals and "supermolecule" calculation on $T_{1}$ achieve MADs of $9.4 \mathrm{~kJ} / \mathrm{mol}$ and $6.2 \mathrm{~kJ} / \mathrm{mol}$, respectively, to these 8 ATcT values. Both methods deliver a systematic underestimation, as is found to be typical of DFT methods. ${ }^{35}$ The MADs we report for B2GP-PLYP, however, are more comparable to those found for the composite CBS-Q and G3 methods, than those of conventional DFT results. ${ }^{35}$

In general, the two protocols deliver similar asymptotic values, with a MAD of $2.5 \mathrm{~kJ} / \mathrm{mol}$ between the theoretical estimates, with the "supermolecule" protocol slightly more accurate with respect to the ATcT values. The MaxAE of $14.9 \mathrm{~kJ} / \mathrm{mol}$ was obtained for the "separate" calculation of $E^{\text {asymptotic }}$ of acetone. Notably only one calculation, the "supermolecule" 
Table S3 Asymptotic $\Delta H_{\text {react }}(0 \mathrm{~K})$ for NTI ground state radical products calculated using the separate radical (Separate) and supermolecule (Supermol.) computational protocols (see text) and from Active Thermochemical Tables (ATcT) $0 \mathrm{~K}$ heats of formation. All energies in $\mathrm{kJ} / \mathrm{mol}$.

\begin{tabular}{|c|c|c|c|c|c|c|}
\hline & \multicolumn{3}{|c|}{ NTI $a$ dissociation } & \multicolumn{3}{|c|}{ NTI $b$ dissociation } \\
\hline & Separate & Supermol. & $\mathrm{ATcT}^{a}$ & Separate & Supermol. & $\mathrm{ATcT}^{a}$ \\
\hline \multicolumn{7}{|l|}{ Aldehydes: } \\
\hline Formaldehyde $^{b}$ & 351 & 354 & $362.8 \pm 0.1$ & & & \\
\hline Acetaldehyde & 338 & 344 & $346.2 \pm 0.3$ & 357 & 357 & $367.7 \pm 0.5$ \\
\hline Propanal & 332 & 335 & $339.6 \pm 2.5$ & 356 & 356 & - \\
\hline Butanal & 340 & 341 & - & 360 & 360 & - \\
\hline Pentanal & 341 & 345 & - & 361 & 362 & - \\
\hline 2-Methylpropanal & 328 & 332 & $330.7 \pm 2.6$ & 359 & 358 & - \\
\hline Pivaldehyde & 324 & 329 & - & 358 & 358 & - \\
\hline \multicolumn{7}{|l|}{ Ketones: } \\
\hline Acetone $^{b}$ & 331 & 335 & $345.9 \pm 0.5$ & & & \\
\hline Butanone & 337 & 338 & - & 343 & 346 & - \\
\hline Pentan-2-one & 336 & 338 & - & 338 & 342 & - \\
\hline Pentan-3-one ${ }^{b}$ & 341 & 342 & - & & & \\
\hline \multicolumn{7}{|c|}{$\alpha, \beta$-Unsaturated carbonyls: } \\
\hline Acrolein & 387 & 393 & - & 351 & 352 & - \\
\hline Crotonaldehyde & 409 & 412 & - & 362 & 363 & - \\
\hline Methacrolein & 377 & 381 & - & 362 & 363 & - \\
\hline MVK & 389 & 394 & - & 335 & 338 & - \\
\hline MIPK & 374 & 376 & - & 340 & 343 & - \\
\hline \multicolumn{7}{|l|}{ Dicarbonyls: } \\
\hline Glyoxal & 279 & 284 & $289.8 \pm 0.5$ & 348 & 348 & - \\
\hline Methylglyoxal & 289 & 293 & - & 355 & 354 & - \\
\hline Diacetyl & 295 & 298 & $303.3 \pm 0.9$ & 346 & 346 & - \\
\hline \multicolumn{7}{|l|}{ Carbohydrates: } \\
\hline Glycolaldehyde & 304 & 310 & - & 357 & 358 & - \\
\hline
\end{tabular}

${ }^{a}$ a dash $(-)$ indicates ATcT data was not available.

${ }^{b} \mathrm{NTI} a$ and NTI $b$ are equivalent for symmetrically substituted carbonyls.

calculation of the NTI $a$ asymptotic energy of 2-methylpropanal, lies within the experimental uncertainty. Counterpoise corrections did not reduce the MAD of "separate" calculations, and we conclude the def2-TZVP basis set is large enough to effectively describe the relevant systems.

In the absence of additional experimental data, Table S3 indicates the "supermolecular" protocol is more accurate. The separate optimisation of $D_{0}$ radical products, however, is more computationally efficient with only a small loss of accuracy. 


\section{S8. Additional 0-0 Electronic Excitation Energies, NTI Photolysis Thresholds and Intrinsic Barriers.}

Because of the availability of analytic gradients, the B3LYP ${ }^{36,37}$ and CAM-B3LYP 38 density functionals, rather than B2GP-PLYP, were used in TD-DFT optimisations on $S_{1}$. The energies and ZPVE corrections obtained allowed $S_{1}$ intrinsic reaction barriers and, together with $b t$-PNO-STEOM-CCSD ${ }^{39}$ calculations, CC/DFT" vertical and $0-0 S_{0} \rightarrow S_{1}$ excitation energies and photolysis thresholds to be determined. Results obtained using B2GP-PLYP $S_{0}$ calculations, $b t$-PNO-STEOM-CCSD vertical excitation energies and TD-B3LYP $S_{1}$ calculations are given in the main text. Corresponding CAM-B3LYP 0-0 excitation energies are shown in Table S4, denoted CC/CAM-B3LYP. For reference, 0-0 excitation energies have also been calculated solely using TD-B3LYP (and B3LYP $S_{0}$ calculations) and solely using TD-CAM-B3LYP (and CAM-B3LYP $S_{0}$ calculations). These are denoted TD-B3LYP and TD-CAM-B3LYP, respectively, in Table S4. Table S4 also gives CC/CAM-B3LYP photolysis thresholds and TD-CAM-B3LYP intrinsic barrier heights for NTI $a$ and NTI $b$ reaction on $S_{1}$. The TD-CAM-B3LYP intrinsic barrier heights for NTI $a$ and NTI $b$ reaction on $S_{1}$, are also shown in Figure S2, with the equivalent TD-B3YP results reproduced from than main text as open symbols.

From Table S4, the composite CC/DFT" scheme is significantly more accurate, with respect to the available experimental 0-0 excitation energies, than either TD-B3LYP or TDCAM-B3LYP. Comparison to Table 1 in the main text and Table S4 shows that the CC/B3LYP results yield slightly lower MAD and MaxAE than the CC/CAM-B3LYP calculations, that is, the CC/B3LYP results are slightly closer to the available experimental data.

Figure S2 shows that the CAM-B3LYP intrinsic barrier heights are systematically higher than those calculated at B3LYP. The trends within and between carbonyl class, however, are the same. 
Table S4 Additional results for 0-0 electronic excitation energies as indicated (see text), CC/CAM-B3LYP NTI photolysis thresholds and TD-CAM-B3LYP NTI intrinsic barriers. Experimental 0-0 excitation energies (Exp) compiled in Godunov et al. ${ }^{52}$ are also included, together with mean absolute deviations (MAD) and maximum absolute error (MaxAE) with respect to experiment. All energies in $\mathrm{kJ} / \mathrm{mol}$.

\begin{tabular}{|c|c|c|c|c|c|c|c|c|}
\hline & \multicolumn{4}{|c|}{ 0-0 Electronic Excitation Energy } & \multicolumn{2}{|c|}{ Photolysis Threshold } & \multicolumn{2}{|c|}{ Intrinsic Barrier } \\
\hline & \multicolumn{4}{|c|}{$S_{0} \rightarrow S_{1}$} & NTI $a$ & NTI $b$ & NTI $a$ & $\mathrm{NTI} b$ \\
\hline & Exp. $^{a}$ & TD-B3LYP. & CC/CAM-B3LYP" & TD-CAM-B3LYP & $S_{1}$ & $S_{1}$ & $S_{1}$ & $S_{1}$ \\
\hline Aldehydes: & & & & & & & & \\
\hline Formaldehyde $^{b}$ & $337^{40}$ & 353 & 358 & 367 & 497 & & 139 & \\
\hline Acetaldehyde & $356^{41}$ & 371 & 370 & 387 & 462 & 503 & 92 & 133 \\
\hline Propanal & $350^{42}$ & 366 & 366 & 383 & 445 & 499 & 79 & 133 \\
\hline Butanal & - & 370 & 367 & 387 & 448 & 498 & 81 & 131 \\
\hline Pentanal & - & 370 & 367 & 386 & 445 & 491 & 83 & 129 \\
\hline 2-Methylpropanal & $349^{43}$ & 367 & 368 & 389 & 428 & 504 & 60 & 136 \\
\hline Pivaldehyde $^{c}$ & $349^{44}$ & 367 & 362 & 384 & 419 & - & 53 & - \\
\hline Ketones: & & & & & & & & \\
\hline Acetone $^{b}$ & $364^{45}$ & 367 & 371 & 395 & 490 & & 119 & \\
\hline Butanone & $359^{46}$ & 378 & 369 & 397 & 465 & 484 & 96 & 115 \\
\hline Pentan-2-one & - & 372 & 367 & 391 & 462 & 482 & 95 & 115 \\
\hline Pentan-3-one ${ }^{b}$ & - & 379 & 373 & 398 & 466 & & 93 & \\
\hline$\alpha, \beta$-Unsaturated & arbonyls. & & & & & & & \\
\hline Acrolein & $310^{47}$ & 294 & 317 & 330 & 484 & 440 & 167 & 123 \\
\hline Crotonaldehyde & $317^{48}$ & 315 & 337 & 357 & 503 & 452 & 166 & 115 \\
\hline Methacrolein & $317^{48}$ & 304 & 323 & 338 & 462 & 455 & 139 & 132 \\
\hline $\mathrm{MVK}^{c}$ & $313^{48}$ & 297 & 319 & 336 & 507 & - & 188 & - \\
\hline MIPK & - & 307 & 321 & 343 & 476 & 438 & 155 & 117 \\
\hline Dicarbonyls: & & & & & & & & \\
\hline Glyoxal $^{c}$ & $262^{49}$ & 230 & 267 & 265 & 410 & - & 143 & - \\
\hline Methylglyoxal $^{c}$ & $265^{50}$ & 239 & 265 & 268 & 411 & - & 146 & - \\
\hline Diacetyl $^{c}$ & $265^{51}$ & 242 & 261 & 270 & - & - & - & - \\
\hline $\begin{array}{l}\text { Carbohydrates: } \\
\text { Glycolaldehyde }\end{array}$ & - & 345 & 345 & 362 & 410 & 476 & 65 & 131 \\
\hline MAD vs. Exp. & & 16.8 & 10.6 & 25.3 & & & & \\
\hline MaxAE vs. Exp. & & 25 & 22 & 40 & & & & \\
\hline
\end{tabular}

${ }^{a}$ A dash (-) indicates no 0-0 excitation energy reported in Reference 52.

${ }^{b}$ NTI $a$ and NTI $b$ are equivalent for symmetrically substituted carbonyls.

${ }^{c}$ first order saddle points for $S_{1}$ NTI $b$ reaction could not be located. 


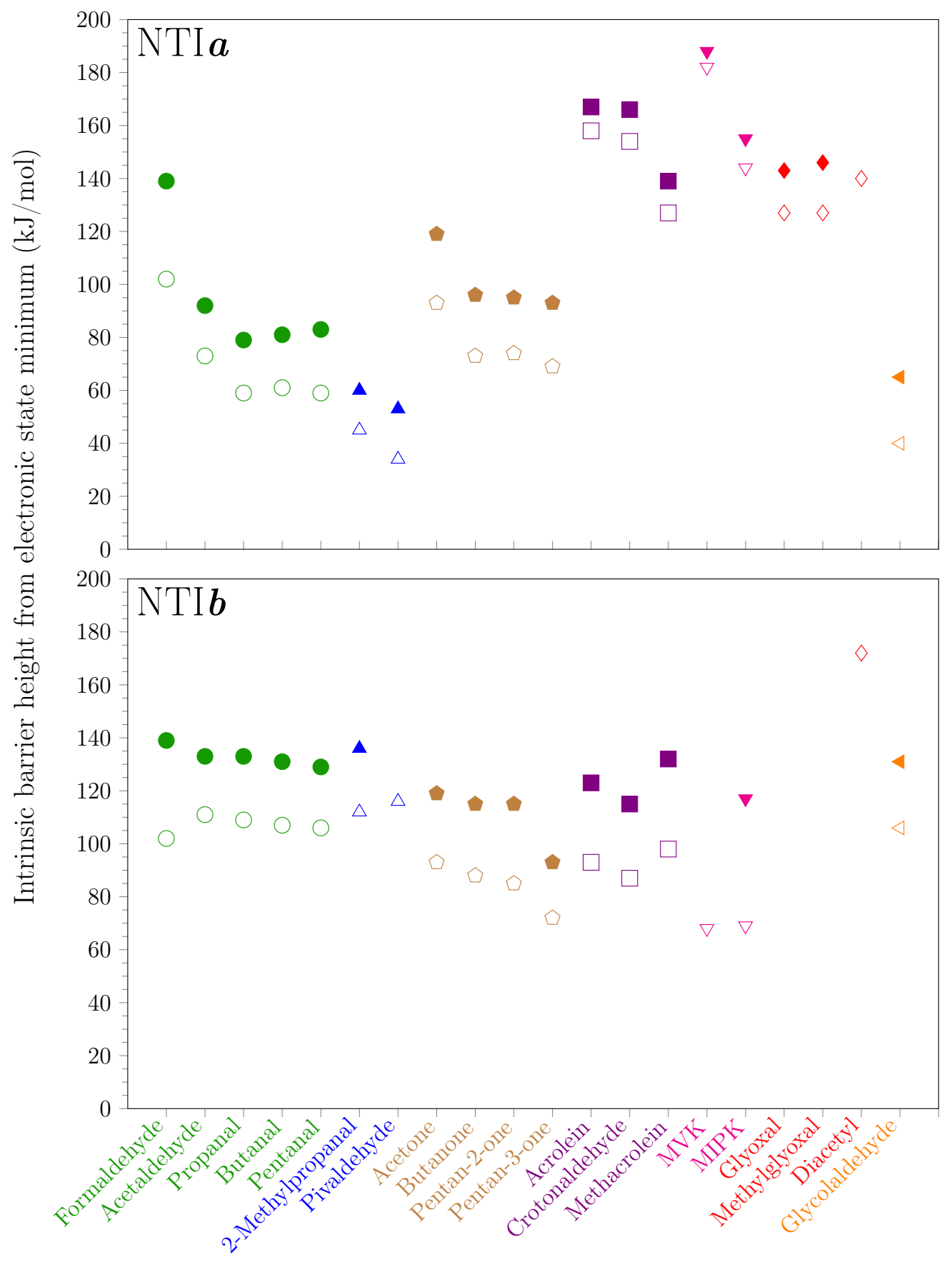

Figure S2: CAM-B3LYP calculated intrinsic barrier heights of $S_{1}$ NTI $a$ (upper panel) and $S_{1}$ NTIb (lower panel) reactions relative to the $S_{1}$ excited state minima. CAM-B3LYP are those with filled symbols $(\bullet)$, while B3LYP results are shown in unfilled symbols $(\bigcirc)$ for comparison. Symbol shape and colour indicate carbonyl classes: linear aldehydes $(\bullet)$, branched aldehydes $(\mathbf{\Delta})$, linear ketones $(\bullet)$, enals $(\boldsymbol{\nabla})$, enones $(\boldsymbol{\nabla})$, dicarbonyls $(\triangleleft)$, and carbohydrates $(\triangleleft)$. Note that NTI intrinsic barriers for the three symmetric carbonyls have been included in both panels. First order saddle-points could not be located using CAM-B3LYP/def2-TZVP for the $S_{1} \mathrm{NTI} b$ reactions of diacetyl, glyoxal, methylglyoxal, MVK, and pivaldehyde. 


\section{S9. Calculated and experimental geometries}

Experimental $S_{0}$ ground state bond lengths ${ }^{53-56}$ are compared to our B2GP-PLYP calculations for the minimum energy geometries of formaldehyde, acetaldehyde, propanal and acetone in Table S5. Comparison to the CCSD(T)/aug-cc-pVQZ geometries of formaldehyde, acetaldehyde and glyoxal from the W4-17 dataset $^{57}$ are shown in Table S6. The notation in these tables sets the aldehyde carbon as atom number ' 1 ', the oxygen as ' 2 ' and the formyl $\mathrm{H}$ as '3'. Each $\mathrm{CH}_{n}$ group is then labelled sequentially. In acetone, the $\mathrm{C}(\mathrm{C}=\mathrm{O}) \mathrm{C}$ atoms are labelled 1, 2, 3 and 4, respectively.

Table S5 Comparison between B2GP-PLYP/def2-TZVP and experimental $S_{0}$ bond lengths for selected carbonyls.

\begin{tabular}{|c|c|c|c|c|c|c|c|}
\hline & \multicolumn{3}{|c|}{ Bond length $(\AA)$} & & \multicolumn{3}{|c|}{ Bond length $(\AA)$} \\
\hline & Theory & Expt. $^{a}$ & Diff. & & Theory & Expt. $^{a}$ & Diff. \\
\hline Formaldehyde & & & & Acetone & & & \\
\hline $\mathrm{C}^{1}-\mathrm{O}^{2}$ & 1.20107 & 1.205 & 0.004 & $\mathrm{C}^{2}-\mathrm{O}^{3}$ & 1.2095 & 1.215 & 0.006 \\
\hline $\mathrm{C}^{1}-\mathrm{H}^{3}$ & 1.10143 & 1.11101 & 0.010 & $\mathrm{C}^{2}-\mathrm{C}^{1}$ & 1.50757 & 1.515 & 0.007 \\
\hline $\mathrm{C}^{1}-\mathrm{H}^{4}$ & 1.10143 & 1.11101 & 0.010 & $\mathrm{C}^{2}-\mathrm{C}^{4}$ & 1.50764 & 1.515 & 0.007 \\
\hline Mean & & & 0.008 & $\mathrm{C}^{1}-\mathrm{H}^{5}$ & 1.08468 & 1.086 & 0.001 \\
\hline Acetaldehyde & & & & $\mathrm{C}^{1}-\mathrm{H}^{6}$ & 1.08988 & 1.086 & 0.004 \\
\hline $\mathrm{C}^{1}-\mathrm{O}^{2}$ & 1.20441 & 1.216 & 0.012 & $\mathrm{C}^{1}-\mathrm{H}^{7}$ & 1.0899 & 1.086 & 0.004 \\
\hline $\mathrm{C}^{1}-\mathrm{H}^{3}$ & 1.10603 & 1.114 & 0.008 & $\mathrm{C}^{4}-\mathrm{H}^{8}$ & 1.08467 & 1.086 & 0.001 \\
\hline $\mathrm{C}^{1}-\mathrm{C}^{4}$ & 1.49639 & 1.501 & 0.005 & $\mathrm{C}^{4}-\mathrm{H}^{9}$ & 1.08979 & 1.086 & 0.004 \\
\hline $\mathrm{C}^{4}-\mathrm{H}^{5}$ & 1.08522 & 1.086 & 0.001 & $\mathrm{C}^{4}-\mathrm{H}^{10}$ & 1.08999 & 1.086 & 0.004 \\
\hline $\mathrm{C}^{4}-\mathrm{H}^{6}$ & 1.09054 & 1.086 & 0.005 & Mean & & & 0.004 \\
\hline $\mathrm{C}^{4}-\mathrm{H}^{7}$ & 1.09054 & 1.086 & 0.005 & & & & \\
\hline Mean & & & 0.005 & & & & \\
\hline Propanal & & & & & & & \\
\hline $\mathrm{C}^{1}-\mathrm{O}^{2}$ & 1.20463 & 1.210 & 0.005 & & & & \\
\hline $\mathrm{C}^{1}-\mathrm{H}^{3}$ & 1.10804 & 1.115 & 0.007 & & & & \\
\hline $\mathrm{C}^{1}-\mathrm{C}^{4}$ & 1.49962 & 1.509 & 0.009 & & & & \\
\hline $\mathrm{C}^{4}-\mathrm{H}^{5}$ & 1.08765 & 1.105 & 0.017 & & & & \\
\hline $\mathrm{C}^{4}-\mathrm{H}^{7}$ & 1.09274 & 1.105 & 0.012 & & & & \\
\hline $\mathrm{C}^{4}-\mathrm{C}^{6}$ & 1.52761 & 1.523 & 0.005 & & & & \\
\hline $\mathrm{C}^{6}-\mathrm{H}^{8}$ & 1.08824 & 1.103 & 0.015 & & & & \\
\hline $\mathrm{C}^{6}-\mathrm{H}^{9}$ & 1.08903 & 1.103 & 0.014 & & & & \\
\hline $\mathrm{C}^{6}-\mathrm{H}^{10}$ & 1.08717 & 1.096 & 0.009 & & & & \\
\hline Mean & & & 0.009 & & & & \\
\hline
\end{tabular}

${ }^{a}$ Experimental data: Formaldehyde, ${ }^{53}$ acetaldehyde,,${ }^{54}$ propanal ${ }^{55}$ and acetone. ${ }^{56}$

The mean deviations of the calculated and experimental bond lengths indicate that the B2GP-PLYP geometries accurately describe the experimental molecules. In almost all cases 
Table S6 Comparison between B2GP-PLYP/def2-TZVP and CCSD(T)/aug-cc-pVQZ $S_{0}$ equilibrium bond lengths for the carbonyls in the W4-17 dataset. ${ }^{57}$

\begin{tabular}{|c|c|c|c|c|c|c|c|}
\hline & \multicolumn{3}{|c|}{ Bond length $(\AA)$} & & \multicolumn{3}{|c|}{ Bond length $(\AA)$} \\
\hline & B2GP-PLYP & $\operatorname{CCSD}(\mathrm{T})$ & Diff. & & B2GP-PLYP & CCSDT & Diff. \\
\hline Formaldehyde & & & & Glyoxal & & & \\
\hline $\mathrm{C}^{1}-\mathrm{O}^{2}$ & 1.20107 & 1.2066 & 0.006 & $\mathrm{C}^{1}-\mathrm{O}^{2}$ & 1.2028 & 1.20741 & 0.005 \\
\hline $\mathrm{C}^{1}-\mathrm{H}^{3}$ & 1.10143 & 1.1022 & 0.001 & $\mathrm{C}^{1}-\mathrm{H}^{4}$ & 1.10165 & 1.10252 & 0.001 \\
\hline $\mathrm{C}^{1}-\mathrm{H}^{4}$ & 1.10143 & 1.1022 & 0.001 & $\mathrm{C}^{2}-\mathrm{C}^{4}$ & 1.51589 & 1.51926 & 0.003 \\
\hline Mean & & & 0.002 & $\mathrm{C}^{1}-\mathrm{H}^{4}$ & 1.2028 & 1.20741 & 0.005 \\
\hline Acetaldehyde & & & & $\mathrm{C}^{4}-\mathrm{O}^{5}$ & & & \\
\hline $\mathrm{C}^{1}-\mathrm{O}^{2}$ & 1.20441 & 1.2088 & 0.004 & $\mathrm{C}^{4}-\mathrm{O}^{6}$ & 1.10165 & 1.10252 & 0.001 \\
\hline $\mathrm{C}^{1}-\mathrm{H}^{3}$ & 1.10603 & 1.10674 & 0.001 & Mean & & & 0.003 \\
\hline $\mathrm{C}^{1}-\mathrm{C}^{4}$ & 1.49639 & 1.5025 & 0.006 & & & & \\
\hline $\mathrm{C}^{4}-\mathrm{H}^{5}$ & 1.08522 & 1.08737 & 0.002 & & & & \\
\hline $\mathrm{C}^{4}-\mathrm{H}^{6}$ & 1.09054 & 1.09232 & 0.002 & & & & \\
\hline $\mathrm{C}^{4}-\mathrm{H}^{7}$ & 1.09054 & 1.09232 & 0.002 & & & & \\
\hline Mean & & & 0.002 & & & & \\
\hline
\end{tabular}

experimental bond lengths are larger than the calculated bond lengths, as would be expected from anharmonicity. The mean bond length deviations between the B2GP-PLYP/def2-TZVP and CCSD(T)/aug-cc-pVQZ calculations are also small, demonstrating agreement with high level theoretical methods, as shown in Table S6. Optimisations at both the RI-B2GP-PLYP and B2GP-PLYP levels of theory were performed, with structures almost identical - deviations were $\sim 0.001 \AA$.

There is little available experimental data for the geometries of electronically excited carbonyls. Godunov et al. ${ }^{52}$ have compiled $\mathrm{C}=\mathrm{O}$ distances for the ground and $S_{1}$ excited states of selected carbonyls and these are compared to our DFT predictions in Table S7. Although larger discrepancies are seen between calculated and experimental $\mathrm{C}=\mathrm{O}$ distances for the $S_{1}$ state, there is likely considerable anharmonicity (and uncertainty) in the experimental values. 
Table S7 Comparison between calculated $\mathrm{C}=\mathrm{O}$ bond lengths and those compiled in Godunov et al. ${ }^{52}$

\begin{tabular}{|c|c|c|c|c|c|c|c|c|}
\hline & \multicolumn{3}{|c|}{$S_{0} \mathrm{r}(\mathrm{C}=\mathrm{O})(\AA)$} & \multicolumn{5}{|c|}{$S_{1} \mathrm{r}(\mathrm{C}=\mathrm{O})(\AA)$} \\
\hline & Expt. & B2GP-PLYP & Diff. & Expt. & TD-B3LYP & Diff. & TD-CAM-B3LYP & Diff. \\
\hline Formaldehyde & 1.203 & 1.20107 & 0.0019 & 1.323 & 1.29349 & 0.0295 & 1.28141 & 0.04159 \\
\hline Acetaldehyde & 1.216 & 1.20441 & 0.0116 & 1.316 & 1.30037 & 0.0156 & 1.28722 & 0.02878 \\
\hline Acetone & 1.222 & 1.2095 & 0.0125 & 1.32 & 1.30692 & 0.0131 & 1.29307 & 0.02693 \\
\hline Propanal (cis) & 1.21 & 1.20391 & 0.0061 & 1.31 & 1.29643 & 0.0136 & - & - \\
\hline Propanal (gauche) & 1.21 & 1.20463 & 0.0054 & 1.31 & 1.29679 & 0.0132 & 1.28441 & 0.02559 \\
\hline 2-Methylpropanal (gauche) & 1.209 & 1.2046 & 0.0044 & 1.309 & 1.29184 & 0.0172 & 1.28925 & 0.01975 \\
\hline 2-Methylpropanal (trans) & 1.209 & 1.2045 & 0.0045 & 1.309 & 1.29093 & 0.0181 & - & - \\
\hline Pivaldehyde & 1.21 & 1.20395 & 0.0060 & 1.31 & 1.28684 & 0.0232 & 1.27625 & 0.03375 \\
\hline Acrolein (trans) & 1.215 & 1.2083 & 0.0067 & 1.332 & 1.28071 & 0.0513 & 1.2761 & 0.0559 \\
\hline Crotonaldehyde (trans) & 1.215 & 1.21003 & 0.0050 & 1.34 & 1.28711 & 0.0529 & 1.28256 & 0.05744 \\
\hline Methacrolein (trans) & 1.215 & 1.20898 & 0.0060 & 1.345 & 1.28593 & 0.0591 & 1.27794 & 0.06706 \\
\hline Methyl vinyl ketone (trans) & 1.215 & 1.21497 & 0.0000 & 1.358 & 1.28401 & 0.0740 & - & - \\
\hline Methyl vinyl ketone (trans) & 1.215 & 1.21497 & 0.0000 & 1.358 & 1.28401 & 0.0740 & 1.28088 & 0.07712 \\
\hline Glyoxal (trans) & 1.202 & 1.2028 & 0.0008 & - & 1.22724 & - & - & - \\
\hline Glyoxal (cis) & 1.21 & 1.19832 & 0.0117 & 1.25 & 1.22321 & 0.0268 & 1.21779 & 0.03221 \\
\hline Mean & & & 0.006 & & & 0.034 & & 0.042 \\
\hline
\end{tabular}

All methods are using RI, and the def2-TZVP basis-set.

\section{References}

(1) El-Sayed, M. A. Spin-Orbit Coupling and the Radiationless Processes in Nitrogen Heterocyclics. J. Chem. Phys. 1963, 38, 2834-2838.

(2) Plasser, F.; Wormit, M.; Dreuw, A. New Tools for the Systematic Analysis and Visualization of Electronic Excitations. I. Formalism. J. Chem. Phys. 2014, 141, 024106.

(3) Goerigk, L.; Grimme, S. Double-Hybrid Density Functionals. Wiley Interdiscip. Rev.: Comput. Mol. Sci. 2014, 4, 576-600.

(4) Grimme, S. Semiempirical Hybrid Density Functional with Perturbative Second-Order Correlation. J. Chem. Phys. 2006, 124, 034108.

(5) Grimme, L. G.; S, Efficient and Accurate Double-Hybrid-Meta-GGA Density FunctionalsEvaluation with the Extended GMTKN30 Database for General Main Group Thermochemistry, Kinetics, and Noncovalent Interactions. 2011, 291-309.

(6) Grimme, S.; Antony, J.; Ehrlich, S.; Krieg, H. A Consistent and Accurate Ab Initio Parametrization of Density Functional Dispersion Correction (DFT-D) for the 94 Elements H-Pu. J. Chem. Phys. 2010, 132, 154104.

(7) Neese, F.; Wennmohs, F.; Hansen, A.; Becker, U. Efficient, Approximate and Parallel HartreeFock and Hybrid DFT Calculations. A 'Chain-of-Spheres' Algorithm for the Hartree-Fock Exchange. Chem. Phys. 2009, 356, 98-109.

(8) Weigend, F.; Ahlrichs, R. Balanced Basis Sets of Split Valence, Triple Zeta Valence and Quadruple Zeta Valence Quality for H to Rn: Design and Assessment of Accuracy. Phys. Chem. Chem. Phys. 2005, 7, 3297-305.

(9) Hobday, N.; Quinn, M. S.; Nauta, K.; Andrews, D. U.; Jordan, M. J. T.; Kable, S. H. Experimental and Theoretical Investigation of Triple Fragmentation in the Photodissociation Dynamics of $\mathrm{H}_{2}$ CO. 2013, 117, 12091-12103.

(10) Robert Huber, J. Comment on Chem. Phys. Lett. 371 (2003) 568: Barrier Height for Dissociation 
of Acetaldehyde, $\mathrm{CH}_{3} \mathrm{CHO} \rightarrow \mathrm{CH}_{3}+\mathrm{HCO}$, in the Triplet State T1. Chem. Phys. Lett. 2003, 37r, 481-482.

(11) Harrison, A. W.; Kable, S. H. Photodissociation Dynamics of Propanal and Isobutanal: The Norrish Type I Pathway. J. Chem. Phys. 2018, 148, 164308.

(12) Zuckermann, H.; Schmitz, B.; Haas, Y. Dissociation Energy of an Isolated Triplet Acetone Molecule. J. Phys. Chem. 1988, 92, 4835-4837.

(13) Chen, M.-W.; Lee, S. J.; Chen, I.-C. State-Resolved Dissociation Dynamics of Glyoxal near the Threshold for Formation of Fragment HCO. J. Chem. Phys. 2003, 8347-8355.

(14) Bowman, J. M.; Zhang, X. New Insights on Reaction Dynamics from Formaldehyde Photodissociation. Phys. Chem. Chem. Phys. 2006, 8, 321-332.

(15) Setokuchi, O.; Matuzawa, S.; Shimizu, Y. MRMP Study of Radical Photodissociation Reaction Thresholds for Acetaldehyde and Acetone. Chem. Phys. Lett. 1998, 284, 19-23.

(16) Shaw, M. F. Photochemical Formation of Enols from Carbonyls. Ph.D. thesis, University of Sydney, 2018.

(17) Kletskii, M. E.; Lisovin, A. V.; Burov, O. N.; Kurbatov, S. V. Competing Mechanisms of Norrish and Norrish-Like Reactions in a Wide Range of Systems - from Carbonyl Compounds to Nitrogen Oxide Donators. Comput. Theor. Chem. 2014, 1047, 55-66.

(18) Maeda, S.; Ohno, K.; Morokuma, K. A Theoretical Study on the Photodissociation of Acetone: Insight into the Slow Intersystem Crossing and Exploration of Nonadiabatic Pathways to the Ground State. J. Phys. Chem. Lett. 2010, 1, 1841-1845.

(19) Nádasdi, R.; Zügner, G. L.; Farkas, M.; Dóbé, S.; Maeda, S.; Morokuma, K. Photochemistry of Methyl Ethyl Ketone: Quantum Yields and $\mathrm{S}_{1} / \mathrm{S}_{0}$-Diradical Mechanism of Photodissociation. ChemPhysChem 2010, 11, 3883-3895.

(20) Li, Q. S.; Zhang, F.; Fang, W. H.; Yu, J. G. Probing Mechanistic Photochemistry of Glyoxal in the Gas Phase by Ab Initio Calculations of Potential-Energy Surfaces and Adiabatic and Nonadiabatic Rates. J. Chem. Phys. 2006, 124, 054324.

(21) Maeda, S.; Ohno, K.; Morokuma, K. Systematic Exploration of the Mechanism of Chemical Reactions: The Global Reaction Route Mapping (GRRM) Strategy Using the ADDF and AFIR Methods. Phys. Chem. Chem. Phys. 2013, 15, 3683-3701.

(22) Weigend, F. Hartree-Fock Exchange Fitting Basis Sets for H to Rn. J. Comput. Chem. 2008, 29, 167-175.

(23) Hellweg, A.; Hättig, C.; Höfener, S.; Klopper, W. Optimized Accurate Auxiliary Basis Sets for RI-MP2 and RI-CC2 Calculations for the Atoms Rb to Rn. Theor. Chem. Acc. 2007, 587-597.

(24) Dutta, A. K.; Nooijen, M.; Neese, F.; Izsák, R. Automatic Active Space Selection for the Similarity Transformed Equations of Motion Coupled Cluster Method. J. Chem. Phys. 2017, $146,074103$.

(25) Lee, T. J.; Taylor, P. R. A Diagnostic for Determininig the Quality of Single-Reference Electron Correlation Methods. Int. J. Quantum Chem. 1989, 23, 199-207.

(26) Purvis, G. D.; Bartlett, R. J. A Full Coupled-Cluster Singles and Doubles Model: The Inclusion Disconnected Triples. J. Chem. Phys. 1982, 76, 1910-1918.

(27) Watts, J. D.; Gauss, J.; Bartlett, R. J. Coupled-Cluster Methods with Noniterative Triple Excitations for Restricted Open-Shell Hartree-Fock and Other General Single Determinant Reference Functions. Energies and Analytical Gradients. J. Chem. Phys. 1993, 98, 8718-8733.

(28) Rienstra-Kiracofe, J. C.; Allen, W. D.; Schaefer, H. F. $\mathrm{C}_{2} \mathrm{H}_{5}+\mathrm{O}_{2}$ Reaction Mechanism: High-level Ab Initio Characterizations. J. Phys. Chem. A 2000, 104, 9823-9840.

(29) Thomas, L. H.; Kaltsoyannis, N. An Ab Initio Study of the Electronic Structure of $\mathrm{BCl}_{3}^{2+}$ and its Decomposition Pathways. Phys. Chem. Chem. Phys. 2006, 8, 1271-1281. 
(30) Goldsmith, C. F.; Klippenstein, S. J.; Green, W. H. Theoretical Rate Coefficients for Allyl + $\mathrm{HO}_{2}$ and Allyloxy Decomposition. Proc. Combust. Inst. 2011, 33, 273-282.

(31) Fogueri, U. R.; Kozuch, S.; Karton, A.; Martin, J. M. A Simple DFT-Based Diagnostic for Nondynamical Correlation. Theor. Chem. Acc. 2013, 132, 1-9.

(32) Pople, J. A.; Gill, P. M. W.; Handy, N. C. Spin-Unrestricted Character of Kohn-Sham Orbitals for Open-Shell Systems. Int. J. Quantum Chem. 1995, 56, 303-305.

(33) Boys, S. F.; Bernardi, F. The Calculation of Small Molecular Interactions by the Differences of Separate Total Energies. Some Procedures with Reduced Errors. Mol. Phys. 1970, 19, 553-566.

(34) Ruscic, B. Active Thermochemical Tables: Sequential Bond Dissociation Enthalpies of Methane, Ethane, and Methanol and the Related Thermochemistry. J. Phys. Chem. A 2015, 119, 7810-7837.

(35) Feng, Y.; Liu, L.; Wang, J.-T.; Huang, H.; Guo, Q.-X. Assessment of Experimental Bond Dissociation Energies Using Composite Ab Initio Methods and Evaluation of the Performances of Density Functional Methods in the Calculation of Bond Dissociation Energies. J. Chem. Inf. Comput. Sci 2003, 43, 2005-2013.

(36) Becke, A. D. Density-Functional Thermochemistry. III. The Role of Exact Exchange. J. Chem. Phys. 1993, 98, 5648-5652.

(37) Lee, C.; Yang, W.; Parr, R. G. Development of the Colle-Salvetti Correlation-Energy Formula into a Functional of the Electron Density. Phys. Rev. B 1988, 37, 785-789.

(38) Yanai, T.; Tew, D. P.; Handy, N. C. A New Hybrid Exchange-Correlation Functional Using the Coulomb-Attenuating Method (CAM-B3LYP). Chem. Phys. Lett. 2004, 393, 51-57.

(39) Dutta, A. K.; Nooijen, M.; Neese, F.; Izsák, R. Exploring the Accuracy of a Low Scaling Similarity Transformed Equation of Motion Method for Vertical Excitation Energies. J. Chem. Theory Comput. 2018, 14, 72-91.

(40) Clouthier, D. J.; Ramsay, D. A. The Spectroscopy of Formaldehyde and Thioformaldehyde. Ann. Rev. Phys. Chem. 1983, 34, 31-58.

(41) Noble, M.; Lee, E. K. The Jet-Cooled Electronic Spectrum of Acetaldehyde and Deuterated Derivatives at Rotational Resolution. J. Chem. Phys. 1984, 81, 1632-1642.

(42) Alekseev, V. N.; Godunov, I. A. Oscillating Structure of $\mathrm{A}^{1} \mathrm{~A} \leftarrow \tilde{\mathrm{X}}^{1} \mathrm{~A}$ of cis-Propanal Molecule. Zh. Fiz. Khim. 1993, 67, 99-104.

(43) Badavi, M.; Godunov, I. A. $\mathrm{S}_{1} \leftarrow \mathrm{S}_{0}$ Vibronic Spectrum and Structure of 2-Methylpropanal Molecule in $\mathrm{S}_{1}$ State. Zh. Fiz. Khim. 1993, 67, 490-497.

(44) Yakovlev, N. N.; Godunov, I. A. $S_{1} \leftarrow S_{0}$ Vibronic Spectrum and the Structure of 2,2Dimethylpropanal Molecule in the $\mathrm{S}_{1}$ State. Zh. Fiz. Khim. 1993, 67, 1826-1833.

(45) Yakovlev, N. N.; Dyumaeva, I. V.; Godunov, I. A. Analysis of Oscillating Structure of $\widetilde{\mathrm{A}}{ }^{1} \mathrm{~A}_{2} \leftarrow$ $\tilde{\mathrm{X}}{ }^{1} \mathrm{~A}_{1}$ Transitions of Acetone-h6 and Acetone-d6 Molecules. Zh. Fiz. Khim. 1992, 66, 698-705.

(46) Godunov, I. A.; Badavi, M.; Kuznetsova, T. S. Vibronic $\left(\mathrm{S}_{1} \leftarrow \mathrm{S}_{0}\right)$ Absorption-Spectra and Conformation Analysis of Molecules of Carbonyl-Compounds with Ethyl and Isopropyl Substituents. Zh. Fiz. Khim. 1993, 67, 504-507.

(47) Osborne, G. A.; Ramsay, D. A. Near Ultraviolet Absorption Spectra of cis and trans Acrolein and Acrolein-d1. Can. J. Phys. 1973, 51, 1170-1175.

(48) Birge, R. R.; Pringle, W. C.; Leermakers, P. A. Excited-State Geometries of the Singly Substituted Methylpropenals. I. Vibrational-Electronic Analysis of $\mathrm{S}_{1}\left(\mathrm{n}, \pi^{*}\right)$. J. Am. Chem. Soc. 1971, 93, 6715-6726.

(49) Kamei, S.; Okuyama, K.; Abe, H.; Mikami, N.; Ito, M. Mode Selectivity in Intersystem Crossing: Glyoxal, Methylglyoxal, and Biacetyl. J. Phys. Chem. 1986, 90, 93-100.

(50) Gurnick, M.; Chaiken, J.; Benson, T.; McDonald, J. D. Vibrational and Rotational Spectroscopy 
of the First Electronically Allowed Transition of $\alpha$-Dicarbonyls. J. Chem. Phys. 1981, 74, 99-105.

(51) Soulard, L.; Fillaux, F.; Millié, P. Interpretation of the Vibrational Spectra of Methylglyoxal and Biacetyl in their First Singlet Excited Electronic States. Chem. Phys. 1984, 87, 117-123.

(52) Godunov, I. A.; Yakovlev, N. N.; Khimii, Z. S. Experimental Structural and Conformational Studies of Carbonyl Molecules in the Ground and Lower Excited States. J. Struct. Chem. 1995, 36, 238-253.

(53) Gurvich, L. V., Veyts, I. V., Alcock, C. B., Eds. Thermodynamic Properties of Individual Substances, fourth edition ed.; Hemisphere Publishing Co.: New York, 1989; Vol. 2.

(54) H. Hollenstein, H.; Günthard, H. H. Solid State and Gas Infrared Spectra and Normal Coordinate Analysis of 5 Isotopic Species of Acetaldehyde. Spectrochim. Acta Part A 1971, 27, 2027-2060.

(55) Kuchitsu, K., Ed. Landolt-Bornstein: Group II: Atomic and Molecular Physics Volume 21: Structure Data of Free Polyatomic Molecules; Springer-Verlag: Berlin, 1992; Vol. 21.

(56) Kuchitsu, K., Ed. Structure Data of Free Polyatomic Molecules - Basic Data; Springer-Verlag: Berlin, 1992.

(57) Karton, A.; Sylvetsky, N.; Martin, J. M. L. W4-17: A Diverse and High-Confidence Dataset of Atomization Energies for Benchmarking High-Level Electronic Structure Methods. J. Comput. Chem. 2017, 38, 2063-2075. 